\title{
Identification of Pressure Passive Cerebral Perfusion and Its Mediators after Infant Cardiac Surgery
}

\author{
HAIM BASSAN, KIMBERLEE GAUVREAU, JANE W. NEWBURGER, MILES TSUJI, \\ CATHERINE LIMPEROPOULOS, JANET S. SOUL, GENE WALTER, PETER C. LAUSSEN, \\ RICHARD A. JONAS, AND ADRÉ J. DU PLESSIS
}

Departments of Neurology [H.B., M.T., C.L., J.S.S., G.W., A.J.d.P.], Cardiology [K.G., J.W.N., P.C.L.], Anesthesia [P.C.L.], and Cardiac Surgery [R.A.J.], Children's Hospital Boston and Harvard Medical School, Boston, MA 02115; and Department of Biostatistics [K.G.], Harvard School of Public Health, Boston, MA 02115

\begin{abstract}
Cerebrovascular pressure autoregulation (CPA) regulates cerebral blood flow $(\mathrm{CBF})$ in relation to changes in mean arterial blood pressure (MAP). Identification of a pressure-passive cerebral perfusion and the potentially modifiable physiologic factors underlying it has been difficult to achieve in sick infants. We previously validated the near-infrared spectroscopy-derived hemoglobin difference $(\mathrm{HbD})$ signal (cerebral oxyhemoglobin deoxyhemoglobin) as a reliable measure of changes in CBF in animal models. We now sought to determine whether continuous measurements of $\Delta \mathrm{HbD}$ would correlate to middle cerebral artery flow velocity (CBFV), allow identification and quantification of pressure-passive state, and help to delineate potentially modifiable factors. We enrolled 43 infants ( $2 \mathrm{~d}$ to $7 \mathrm{mo}$ old $)$ who were undergoing open cardiac surgery and cardiopulmonary bypass. At 6 and $20 \mathrm{~h}$ after surgery, we measured changes in $\mathrm{HbD}, \mathrm{CBFV}$ (by transcranial Doppler), and MAP at different end-tidal $\mathrm{CO}_{2}$ levels. We assigned a pressure-passive index (PPI) to each study on the basis of the relative duration of significant coherence between $\triangle \mathrm{MAP}$ and $\Delta \mathrm{HbD}$. We found a significant relationship between $\triangle \mathrm{HbD}$ and $\triangle \mathrm{CBFV}$ at both time points. At $6 \mathrm{~h}$ after surgery, we showed high concordance (coherence $>0.5$; PPI $\geq 41 \%$ ) between $\triangle \mathrm{MAP}$ and $\Delta \mathrm{HbD}$, consistent with disturbed CPA in $13 \%$ of infants. End-tidal $\mathrm{CO}_{2}$ values $\geq 40 \mathrm{~mm} \mathrm{Hg}$ and higher MAP variability both were associated with increased
\end{abstract}

\section{ABSTRACT}

odds $(p<0.001)$ of autoregulatory failure. This approach provides a means to identify and quantify disturbances of CPA. High $\mathrm{CO}_{2}$ levels and fluctuating MAP are two important preventable factors associated with disturbed CPA. (Pediatr Res 57: 35-41, 2005)

$\quad$ Abbreviations
CBF, cerebral blood flow
CBFV, cerebral blood flow velocity
CI, confidence interval
CPA, cerebrovascular pressure autoregulation
CV, coefficient of variation
ET-CO, end-tidal $\mathrm{CO}_{2}$
HbD, hemoglobin difference (oxyhemoglobin -
deoxyhemoglobin)
HbT, hemoglobin total (oxyhemoglobin + deoxyhemoglobin)
ICU, intensive care unit
MAP, mean arterial blood pressure
NIRS, near-infrared spectroscopy
OR, odds ratio
PPI, pressure-passive index (\% time pressure passive state)
RI, resistive index
Sao 2 , arterial oxygen saturation
TCD, transcranial Doppler

Recent advances in infant cardiac surgery and critical care medicine have translated into high survival rates for infants with congenital heart disease. As mortality has decreased, the

Received January 4, 2004; accepted July 19, 2004.

Correspondence: Adré J. du Plessis, M.D., Department of Neurology, Fegan 11, Children's Hospital, 300 Longwood Avenue, Boston MA 02115; e-mail: adre.duplessis@childrens.harvard.edu

This study was supported by a grant from the LifeBridge Fund and grant HL 063411 from the National Heart, Lung, and Blood Institute.

DOI: 10.1203/01.PDR.0000147576.84092.F9 clinical focus has shifted to the prevention of perioperative neurologic injury (1). Previous studies have focused primarily on intraoperative cerebral hypoxia-ischemia as a key mechanism for neurologic dysfunction. However, recently it has become clear that multiple potential mechanisms, operating at different times, may be implicated in the pathogenesis of neurologic dysfunction after infant cardiac surgery (2). In the current research, we focused on the early postoperative period because of its high risk for hemodynamic instability and therefore potential for brain injury. 
A complex system of intrinsic cerebral vasoregulatory mechanisms modulates cerebral blood flow (CBF) to maintain adequate oxygen and nutrients for normal brain metabolism. Previous studies by our group demonstrated an impairment in one component of intrinsic cerebral vasoregulation, $\mathrm{CO}_{2}$ vasoreactivity, during the early postoperative period (3). Cerebrovascular pressure autoregulation $(\mathrm{CPA})$ maintains $\mathrm{CBF}$ constant over a range of cerebral perfusion pressures (the "autoregulatory plateau") (4). When CPA fails, changes in blood pressure are associated with parallel changes in CBF, i.e. the cerebral circulation becomes pressure passive.

Despite the potential for hemodynamic instability in the early period after infant cardiac surgery, CPA has not been adequately studied in this setting. This deficiency is due in part to the lack of techniques for the noninvasive and continuous study of cerebral perfusion in infants during this period of intensive care. Previous studies have evaluated CPA with transcranial Doppler (TCD) to measure CBF velocity. However, the TCD technique has several potential limitations, including the difficulty during prolonged studies of maintaining a constant and reproducible insonation angle to the artery of interest. Moreover, this technique quantifies velocity of blood flow but not volume of blood flow. Near-infrared spectroscopy (NIRS) provides another approach for studying cerebral hemodynamics and oxygenation at the bedside of the sick infant. In previous validation studies in animals, we demonstrated a strong correlation between changes in the NIRS measurement of hemoglobin difference ( $\mathrm{HbD}$; i.e. the difference between cerebral oxyhemoglobin and deoxyhemoglobin concentrations) and $\mathrm{CBF}$ as measured by the gold standard radioactive microsphere technique $(5,6)$. Subsequently, we studied CPA in premature infants by measuring the relationship between $\mathrm{HbD}$ and mean arterial blood pressure (MAP) and identified a pressure-passive state in 53\% of those studied (7).

In the current research, our overall aims were to compare measurements of cerebral perfusion made by TCD and NIRS, to define the integrity of CPA in infants after open-heart surgery, and to determine factors associated with a disturbance in CPA in this setting. We hypothesized that NIRS parameters, specifically $\mathrm{HbD}$, would correlate with $\mathrm{CBF}$ velocity by TCD; pressure-passive cerebral perfusion would be identifiable by simultaneous measurements of $\mathrm{HbD}$ and MAP; and higher $\mathrm{CO}_{2}$ levels would be associated with pressure-passive cerebral perfusion.

\section{METHODS}

Patient enrollment. Infants were enrolled consecutively between July 2001 and August 2002 at Children's Hospital Boston. Eligibility criteria included infants who were $\leq 9$ mo of age, had congenital heart disease, underwent corrective cardiac surgery using deep hypothermia and cardiopulmonary bypass, and had a birth weight $>2.25 \mathrm{~kg}$. The exclusion criteria were known dysmorphic or genetic syndrome, known neurologic abnormality before surgery, and previous cardiac surgery. The study was approved by the Institutional Review Board of Children's Hospital Boston, and written informed parental consent was obtained before surgery. A standardized protocol of cardiopulmonary bypass and anesthesia methods was used during the cardiac operation (8).

Physiologic measurements. We measured cerebral oxygenation and hemodynamics with NIRS (NIRO 500; Hamamatsu Photonics, Hamamatsu City,
Japan) a well-described, noninvasive technique for studying cerebral hemodynamics at the bedside of infants who require critical care $(9,10)$. On the basis of modification of the Beer-Lambert law, determination of changes in light absorbance in the brain allows the calculation of changes in concentration (in micromoles per liter) of the chromophores oxyhemoglobin $\left(\mathrm{HbO}_{2}\right)$, deoxyhemoglobin $(\mathrm{Hb})$, and oxidized cytochrome aa $3\left(\mathrm{CytO}_{2}\right)$. The $\mathrm{HbD}$ is derived by subtracting $\mathrm{Hb}$ from $\mathrm{HbO}_{2}$, whereas their summation results in $\mathrm{Hb}$ total $(\mathrm{HbT})$.

We used transcranial Doppler (TCD; SN: KSO10064; Nicolet Biomedical, Madison, WI) to acquire mean middle cerebral artery timed averaged flow velocity (CBFV) over a period of $5 \mathrm{~s}$. We also obtained mean middle cerebral artery resistive indices [RI; (systolic FV - diastolic FV)/systolic FV], a measure of cerebral vascular resistance. We positioned the TCD probe beside the NIRS optodes on the temporal acoustic window and fixed it with an elastic band for insonation at designated times.

We measured systemic parameters, including MAP, from an indwelling arterial catheter via a pressure transducer (Hewlett Packard cardiac monitor $\mathrm{M} 1047 \mathrm{~A}$ ), arterial oxygen saturation $\left(\mathrm{SaO}_{2}\right)$ by pulse oximetry (Nellcor N100, Hayward, $\mathrm{CA}$ ), and end tidal $\mathrm{CO}_{2}\left(\mathrm{ET}-\mathrm{CO}_{2}\right)$ by end tidal capnometer (Novometrix Capnogard, Wallingford, CT).

Study design. We aimed to perform cerebral and systemic measurements at two time points in the postoperative period; $6 \mathrm{~h}$ ("early sessions") and $20 \mathrm{~h}$ ("late sessions") after the onset of intraoperative rewarming from hypothermia. We commenced each study only after the approval of the cardiac intensive care unit (ICU) attending physician. Continuously and simultaneously, we recorded all NIRS parameters and systemic measurements $\left(\mathrm{MAP}, \mathrm{SaO}_{2}\right.$, and $\left.\mathrm{ET}-\mathrm{CO}_{2}\right)$. All recorded data were sampled at a frequency of $2 \mathrm{~Hz}$, displayed and stored in a laptop computer in a time-locked manner.

A period of at least $10 \mathrm{~min}$ of stable $\mathrm{CO}_{2}$ (as monitored by ET- $\mathrm{CO}_{2}$ ) and $\mathrm{HbD}$ (as monitored by NIRS) was recorded. We then induced measured changes in $\mathrm{CO}_{2}$ by adjusting the ventilator rate while maintaining constant ventilatory pressure, inspired $\mathrm{O}_{2}$ concentrations, and $\mathrm{SaO}_{2}$. We then allowed sufficient time for $\mathrm{CO}_{2}$ and $\mathrm{HbD}$ to stabilize at the new $\mathrm{CO}_{2}$ level as assessed by visual inspection of the on-line screen display. ET- $\mathrm{CO}_{2}$ measurements were adjusted to arterial $\mathrm{CO}_{2}$ measurements at the beginning of each study session. Before and after a ventilator change, we measured CBFV and RI by TCD.

Data processing. We then processed the cerebral and systemic data in the following manner. We visually screened for movement artifacts and eliminated these data to ensure high quality of our measurements. Because NIRS measurements are influenced by systemic $\mathrm{SaO}_{2}$ changes, our measurements included only periods of stable $\mathrm{SaO}_{2}$ with $\mathrm{SaO}_{2}$ fluctuations $<5 \%$.

We then obtained the 1-min average for all systemic and cerebral measurements at two time points, before a ventilator change and after the ET-CO2, $\mathrm{HbD}$, and CBFV signals had stabilized at their new level. Change in these variables was determined by comparing the 1-min averaged values before and after the ventilator rate change. The difference between the measurements was presented as " $\Delta$ values" and used for correlations between changes in TCD and NIRS variables.

We studied CPA by evaluating the correlation between continuous measurements of MAP with simultaneous measurements of cerebral $\mathrm{HbD}$, as an index of CBF. We divided the study data into consecutive and continuous 5-min epochs. We then subjected each 5-min epoch to a uniform and consistent computation. CPA is believed to be frequency specific $(11,12)$. Therefore, we analyzed the relationship between MAP and $\mathrm{HbD}$ in a frequency-specific manner, using transfer function analysis in the frequency domain. Transfer function analysis is an established approach for analyzing the relationship between complex and changing physiologic signals $(7,11,12)$ that uses Fourier transformation to compute the statistical correlation of two waveforms (in our study, MAP and $\mathrm{HbD}$ ). In addition, it is known that autonomic control of the circulation occurs at low frequencies (13). We therefore used detection frequencies of $0-0.1 \mathrm{~Hz}$, a bandwidth that corresponds to changes that occur over several minutes and hence of physiologic importance for autoregulatory control (12).

For minimizing the risk of aliasing, the upper bandwidth detection limit (1 $\mathrm{Hz}$ ) was half of the sampling rate $(2 \mathrm{~Hz}$, or a sampling interval of $0.5 \mathrm{~s}$ ), thereby meeting the requirements of the Nyquist theorem. For each 5-min data epoch, the degree of correlation between the MAP and $\mathrm{HbD}$ waveforms, at a given frequency range, can be quantified as a coherence score. A coherence score ranges between 0 (no correlation) and 1.0 (perfect correlation). By convention, coherence scores $>0.5$ were considered to represent significant 
concordance between the waveforms of MAP and $\mathrm{HbD}$ and, in our model, to indicate disturbed pressure autoregulation. Thus, each 5-min epoch could be categorized as either coherent (coherence score between 0.5 and 1, i.e. pressure passive) or noncoherent (coherence score between 0 and 0.5, i.e. pressure autoregulating). We then assigned an overall pressure-passive index (PPI) to each study session by calculating the percentage time of pressure passivity (i.e. number of coherent epochs/total number of epochs in that study session). A PPI of 0 indicates perfect autoregulation, whereas a PPI of 100\% suggests a completely pressure-passive cerebral circulation over the entire study session. Computations were performed by a Matlab software package (Matlab, Natick, MA).

Next we combined the 5-min data epochs of all patients in the study and divided these into two dichotomous groups according to their MAP and $\mathrm{HbD}$ relationship—coherent (pressure passive) epochs versus noncoherent (pressure autoregulating) epochs-for further specific physiologic correlations. We documented the available mean ET- $\mathrm{CO}_{2}$ and MAP values of each time epoch. Because normal MAP differs with age, we used published normative values for each patient according to his or her age at surgery (14). Each MAP value was categorized as normal, elevated ( $>1 \mathrm{SD}$ or $>2 \mathrm{SD}$ above the mean), or decreased $(<1 \mathrm{SD}$ or $2 \mathrm{SD}$ below the mean). We also calculated the MAP coefficient of variation $(\mathrm{CV})$, defined as the SD divided by the mean, for each 5-min epoch as a measure of MAP instability.

Clinical data. To examine the effect of various clinical factors on CPA, we systematically reviewed the patient's medical record. We considered the following as potential risk factors during the pre- and intraoperative periods for subsequent CPA disturbance: cyanotic congenital heart disease (transposition of great arteries and tetralogy of Fallot), preoperative intubation (as a measure of respiratory instability), deep hypothermic circulatory arrest duration $>20$ $\mathrm{min}$, and minimum intraoperative rectal temperature $<18^{\circ} \mathrm{C}$ (i.e. "deep" hypothermia). We considered the effect on CPA of individual risk factors as well as the effect of the sum of risk factors used as a gross index of morbidity.

In addition, we considered the effect on CPA of specific conditions during each study session: time since intraoperative rewarming, core (rectal) temperature, acidosis (any blood $\mathrm{pH}<7.25$ on day of study), and the medications in use. We reviewed and documented all potentially vasoactive medications used during the course of the study period. These were grouped into the following classes: inotropic agents (digoxin, dobutamine, dopamine), paralyzing agents (pancuronium, rocuronium, cisatracurium), sedative-analgesic agents (fentanyl, morphine, chloral hydrate, midazolam, ketamine), and antihypertensive diuretic agents (spironolactone, captopril, alprostadiol, furosemide). We considered the effect on CPA of individual drug classes (as grouped above), as well as the effect of each drug used.

Statistical analysis. Continuous measurements are expressed as mean \pm $\mathrm{SD}$. The Pearson correlation coefficient $(r)$ was used to measure the relationship between changes in Doppler measurements and changes in NIRS parameters. Generalized estimating equation models, which account for the correlation among multiple measurements made on the same patient, were used to calculate $p$ values. Correlations were estimated for early and late studies separately and for all studies combined. The relationship between patient PPIs at the early and late studies was evaluated using the Spearman rank correlation coefficient $\left(r_{\mathrm{s}}\right)$. Comparisons of mean $\mathrm{CO}_{2}$ levels and percentages of epochs with abnormal MAP were performed for pressure-passive versus autoregulating 5-min epochs using generalized estimating equation models to account for the correlation among multiple epochs for the same patient; these models were also used to estimate odds ratios (OR). For each patient, relationships between categories of PPI and potential risk factors were evaluated using Fisher's exact test.

\section{RESULTS}

We studied 43 infants (17 girls), aged $2 \mathrm{~d}$ to 7 mo (median 9 d), who overall had a total of 75 study sessions. After the onset of rewarming, early study sessions occurred after a mean of $5.7 \mathrm{~h}( \pm 1.1)$, and late study sessions occurred after a mean of $19.8 \mathrm{~h}( \pm 2.9)$. Study sessions lasted $52 \mathrm{~min}( \pm 1.5)$ and 53 $\min ( \pm 1.2)$ for early and late studies, respectively. Thirty-two $(75 \%)$ infants had both early and late study sessions, whereas 7 had only early study sessions and 4 had only late study sessions. Twenty-one (48.8\%) infants had transposition of the great arteries, $13(30.2 \%)$ had tetralogy of Fallot, and 9 (20.9\%) had ventricular septal defect. Twenty-one $(48.8 \%)$ infants were intubated before admission to surgery, $1(2.3 \%)$ infant had an Apgar score $<5$ at 5 min, and $1(2.3 \%)$ infant was acidotic on a study day. Median rectal temperatures during the early and late study sessions were $36.5^{\circ} \mathrm{C}(33.7-38)$ and $36.5^{\circ} \mathrm{C}$ (35.5-38.8), respectively. The clinical and demographic data of these infants are presented in Table 1. Clinical characteristics of infants with measurements at a single time point did not differ from those with measurements at both early and late sessions.

Relationship between NIRS parameters and Doppler measurements. We evaluated the correlations between $\triangle \mathrm{CBFV}$, $\triangle \mathrm{RI}$, and $\triangle \mathrm{NIRS}$ measurements. We used data from 32 early and 20 late study sessions that had NIRS and TCD data of adequate quality. We excluded TCD and NIRS data from 7 early and 16 late study sessions because of artifactcontaminated tracings (e.g. movement artifacts, baseline shift) or inability to induce $\mathrm{CO}_{2}$ changes because infants had recovered spontaneous respiration. We found no significant clinical difference between patients with excluded study sessions and those with adequate study sessions. Data are presented in Table 2. Changes in ET- $-\mathrm{CO}_{2}$ had no significant effect on MAP or arterial $\mathrm{SaO}_{2}(r=-0.08, \mathrm{p}=\mathrm{NS}$; and $r=0.02, \mathrm{p}=\mathrm{NS}$, respectively). We found a significant relationship between $\triangle \mathrm{CBFV}$ and $\triangle \mathrm{HbD}$ in both the early and the late studies and in all studies combined $(r=0.64, p<0.001$; Fig. 1$)$. A similar relationship was found between $\Delta \mathrm{HBO}_{2}$ and $\Delta \mathrm{CBFV}(r=$ $0.59, p<0.001)$, whereas the relationship between $\Delta \mathrm{HbT}$ and $\triangle \mathrm{CBFV}$ was marginal $(r=0.32, p=0.04)$. The relationship between $\triangle \mathrm{Hb}$ and $\triangle \mathrm{CBFV}$ was not significant. Furthermore, $\Delta \mathrm{RI}$ showed possible correlations with $\Delta \mathrm{HbD}, \Delta \mathrm{HBO}_{2}$, and $\Delta \mathrm{HbT}(r=-0.37, p=0.006 ; r=-0.41, p<0.001$; and $r=$ $-0.32, p=0.004$, respectively) but not with $\Delta \mathrm{Hb}$. We also found significant relationships between $\Delta \mathrm{CO}_{2}$ and $\Delta \mathrm{HbD}$ and between $\Delta \mathrm{CBFV}$ and $\Delta \mathrm{HbD}, \Delta \mathrm{CBFV}$, and $\Delta \mathrm{RI}(r=0.74, p<$ $0.001 ; r=0.64, p<0.001 ;$ and $r=-0.64, p<0.001$, respectively).

Disturbed CPA at early and late studies. Because the cutoff for defining abnormal PPI is unknown, we arbitrarily divided

Table 1. Clinical data $(n=43)$

\begin{tabular}{lc}
\hline \multicolumn{1}{c}{ Clinical characteristics } & $\begin{array}{c}\text { Median (range) } \\
\text { or } n(\%)\end{array}$ \\
\hline Age at surgery (d) & $9(2-210)$ \\
Patients with age $\leq 30 \mathrm{~d}$ & $24(55.8 \%)$ \\
Gestational age at birth (wk) & $39(35-41.4)$ \\
Patients with cyanotic CHD & $30(70 \%)$ \\
Patients intubated before admission & $21(49 \%)$ \\
Total support time (min) & $108(57-196)$ \\
Patients with any DHCA & $22(51 \%)$ \\
Duration of DHCA (min) & $27(1-59)$ \\
Lowest intraoperative rectal temperature $\left({ }^{\circ} \mathrm{C}\right)$ & $17.9(13.6-29.1)$ \\
Patients with deep hypothermia $\left(\leq 18^{\circ} \mathrm{C}\right)$ & $23(54 \%)$ \\
Length of time intubated $(\mathrm{d})$ & $1.6(0.9-5.8)$ \\
Time in ICU (d) & $4(2-8)$ \\
Total hospital stay $(\mathrm{d})$ & $7(3-27)$
\end{tabular}

CHD, congenital heart disease; DHCA, deep hypothermic circulatory arrest. 
Table 2. Relationships between changes in Doppler and NIRS measurements during early, late, and combined studies

\begin{tabular}{|c|c|c|c|c|c|c|c|c|c|c|}
\hline \multirow[b]{2}{*}{ MCA Doppler parameters } & \multirow[b]{2}{*}{ NIRS parameters } & \multicolumn{3}{|c|}{ Early studies } & \multicolumn{3}{|c|}{ Late studies } & \multicolumn{3}{|c|}{ Combined studies } \\
\hline & & $r$ & $p$ value & $\mathrm{n}$ & $r$ & $p$ value & $\mathrm{n}$ & $r$ & $p$ value & $\mathrm{n}$ \\
\hline$\triangle \mathrm{CBFV}$ & $\Delta \mathrm{HbT}$ & 0.30 & 0.02 & 37 & 0.32 & NS & 34 & 0.32 & 0.04 & 71 \\
\hline \multirow[t]{2}{*}{$\Delta \mathrm{RI}$} & $\Delta \mathrm{HbT}$ & -0.33 & 0.07 & 56 & -0.39 & NS & 48 & -0.32 & 0.004 & 104 \\
\hline & $\Delta \mathrm{HbD}$ & -0.40 & 0.02 & 56 & -0.41 & NS & 48 & -0.37 & 0.006 & 104 \\
\hline
\end{tabular}

MCA, middle cerebral artery; $\mathrm{n}=$ number of induced $\mathrm{CO}_{2}$ changes.

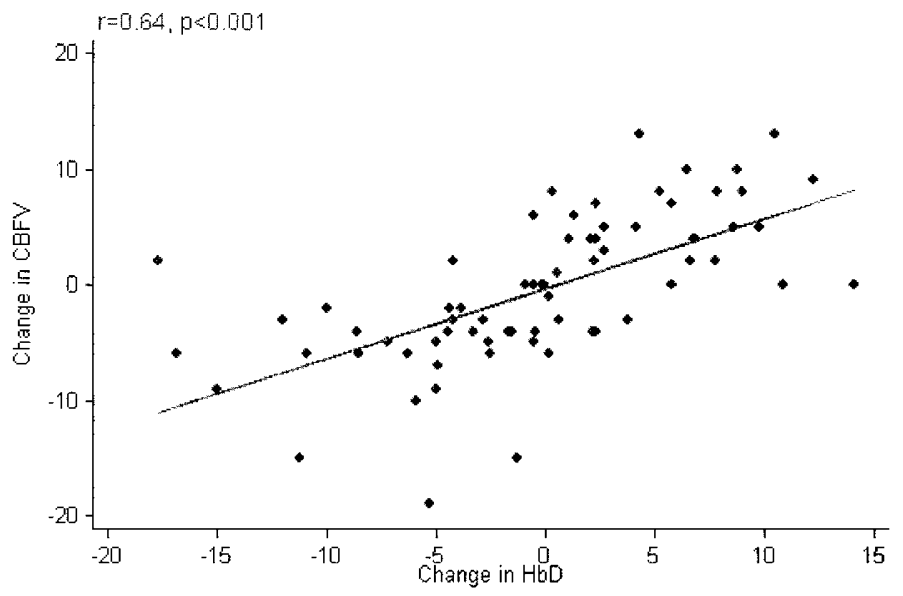

Figure 1. Correlation between $\triangle \mathrm{CBFV}$ and $\triangle \mathrm{HbD}$.

the infants into two groups: intact CPA, i.e. infants who had no concordant relationship between MAP and $\mathrm{HbD}$ (coherence $<0.5$ in all study measurements; PPI $=0$ ), and disturbed CPA, i.e. infants who had periods of a concordant relationship between MAP and $\mathrm{HbD}$ (coherence $>0.5$; PPI $=1-100 \%$ ). Figure 2 shows an example of a patient with disturbed CPA. According to our model, CPA in most infants was either intact or mildly disturbed (PPI 1-20\%). At $6 \mathrm{~h}$ after surgery, we found high concordance (coherence $>0.5$; PPI $\geq 41 \%$ ) between MAP and $\mathrm{HbD}$ in 5 (13\%) infants, consistent with disturbed CPA. Table 3 shows the distribution of PPI for early and late studies separately. For the 32 patients who had both early and late studies, we found a moderate correlation between PPI at each time point $\left(r_{\mathrm{s}}=0.37, p=0.03\right)$. All infants with disturbed CPA (coherence $>0.5$; PPI $\geq 41 \%$ ) at $18 \mathrm{~h}$ had been pressure passive at $6 \mathrm{~h}$. This finding suggests that infants with disturbed CPA during the early postoperative period were likely to remain in a pressure-passive state of CPA into the late postoperative period (Fig. 3).

Data analysis at the epoch level. Acquiring all NIRS and systemic hemodynamic measurements in a continuous timelocked manner allowed an overall data analysis across all epochs. We analyzed data for early and late studies separately because these periods might have different hemodynamic characteristics. Across all 39 patients who had early studies, a total of 359 5-min data epochs were collected. Fifty-three (14.8\%) epochs showed a pressure-passive relationship (i.e. coherence $>0.5$ ) between MAP and HbD, whereas 306 showed an autoregulating relationship (i.e. coherence $<0.5$ ). Across the 36 patients who had late studies, a total of 313 epochs were measured, $43(13.7 \%)$ of which showed a pressure-passive

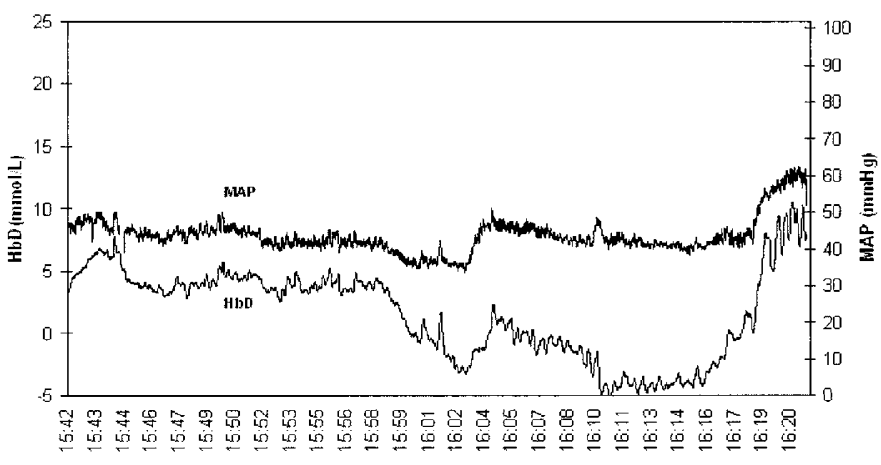

Figure 2. Disturbed cerebral pressure autoregulation. PPI $=62 \%$.

Table 3. Percentage of time spent by patients in state of pressure-passive cerebral perfusion during early and late studies

\begin{tabular}{lcc}
\hline \multicolumn{1}{c}{ PPI } & Early studies [39 patients; & Late studies [36 patients; \\
& $n(\%)]$ & $n(\%)]$ \\
\hline 0 & $20(51 \%)$ & $12(33 \%)$ \\
$1-20 \%$ & $12(31 \%)$ & $16(44 \%)$ \\
$21-40 \%$ & $2(5 \%)$ & $5(14 \%)$ \\
$41-60 \%$ & $3(8 \%)$ & $2(6 \%)$ \\
$\geq 61 \%$ & $2(5 \%)$ & $1(3 \%)$ \\
\hline
\end{tabular}

relationship and 270 of which showed an autoregulating relationship between MAP and $\mathrm{HbD}$. We used the total sets of 5-min data epochs to examine the relation between CPA and ET- $\mathrm{CO}_{2}$ as well as the effect of mean MAP and its variance over each 5-min epoch.

Association between ET-CO levels and CPA. ET- $\mathrm{CO}_{2}$ values were available for 348 of 359 early study epochs. Mean ET- $\mathrm{CO}_{2}$ was $40.9 \pm 5.5$ for pressure-passive epochs and $35.9 \pm$ 5.9 for autoregulating epochs $(p<0.001)$; thus, a higher ET- $\mathrm{CO}_{2}$ was associated with a pressure-passive versus an autoregulating epoch. Having ET- $\mathrm{CO}_{2}$ value $\geq 40$ increased the odds of an epoch's being pressure passive compared with ET- $\mathrm{CO}_{2}<40$ [OR 5.2; 95\% confidence interval (CI) 2.4-10.9; $p<0.001$; Table 4].

ET- $\mathrm{CO}_{2}$ measurements were available for 292 of 313 late study epochs. Mean ET- $\mathrm{CO}_{2}$ was $41.0 \pm 6.3$ for pressurepassive epochs and $39.0 \pm 5.2$ for autoregulating epochs $(p=$ 0.04). However, the odds of an epoch's being pressure passive with ET- $\mathrm{CO}_{2}$ value $\geq 40$ were not statistically significant (OR 1.7; 95\% CI $0.8-3.6 ; p=0.15$ ). The association between hypercapnea and disturbed autoregulation thus is more conspicuous during early studies.

Association between MAP and CPA. Considering all of the 5 -min epochs across all studies, we found that 200 (56\%) early study epochs and 217 (69\%) late study epochs had mean MAP 


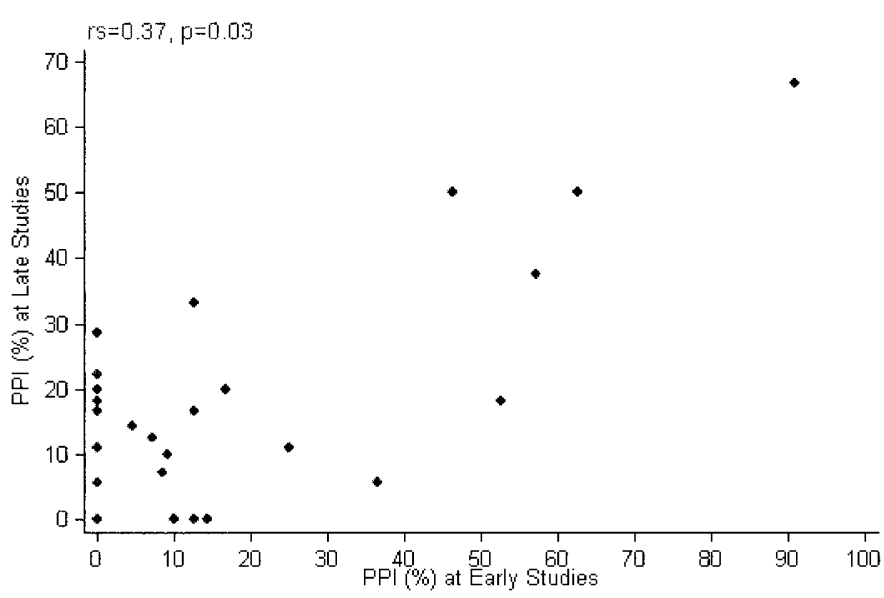

Figure 3. Cerebral pressure autoregulation at early versus late postoperative studies.

values that were considered "normal" for age (see definition in "Methods"). A total of 137 (38\%) early study epochs and 92 (29\%) late study epochs had mean MAP values between 1 and 2 SD above or below the mean. Only $21(6 \%)$ epochs of early studies and $3(1 \%)$ epochs of late studies had MAP values at least $2 \mathrm{SD}$ either above or below the expected normal for age. Although a larger percentage of early study epochs was either low or high, the vast majority of epochs had normal or nearnormal mean MAP values. No significant relationship was found between either low MAP $(<1 \mathrm{SD}$ or $<2 \mathrm{SD}$ below the mean) or high MAP ( $>1$ SD or $>2$ SD above the mean) and an epoch's being pressure passive or autoregulating.

We also calculated the MAP CV of each epoch (see "Methods"). For early studies, MAP CV $\geq 0.08$ increased the odds of an epoch's being pressure passive (OR 4.7; 95\% CI 2.1-10.5; $p<0.001)$. For late studies, MAP CV $\geq 0.08$ again increased the odds of an epoch's being coherent (OR 2.8; 95\% CI $1.6-5.0 ; p<0.001)$.

Association among perioperative risk factors, medication use, and CPA. No significant associations were found between CPA and perioperative risk factors either individually or as a sum of classes. No significant associations were found between CPA and either individual drugs or drug classes (see "Methods" for detailed list of risk factors and drugs).

\section{DISCUSSION}

In this prospective study of infants after cardiac surgery, we first demonstrated a significant correlation between the NIRSderived parameter of $\mathrm{HbD}$ and CBFV measured by TCD. Next we demonstrated the feasibility of using continuous $\mathrm{HbD}$ measurements to evaluate CPA during the early critical care period in these infants. Finally, we demonstrated that CPA may be disturbed in the early postoperative period, particularly at higher $\mathrm{CO}_{2}$ tensions and during periods of fluctuating systemic blood pressure. To our knowledge, the current study is the first to use NIRS measurements and coherence analysis to study CPA in infants after open-heart surgery.

A major impediment to the study of cerebral vasoregulation at the bedside of critically ill infants is the limited availability of safe and reliable techniques for making continuous measurements of cerebral perfusion. Clinical studies have demonstrated the feasibility of using NIRS for measuring cerebral vasoreactivity in critical conditions ranging from deep hypothermic infant cardiac surgery (3) to sick term and preterm infants $(15,16)$. We used in the current study the NIRS-derived $\mathrm{HbD}$ as a measure of $\mathrm{CBF}$, having previously validated this technique in two neonatal piglet models using radioactive microspheres $(5,6)$.

Of interest in our current study is the finding of a significant correlation between measurements of $\Delta \mathrm{HbD}$ and measurements of $\triangle \mathrm{CBFV}$ and $\triangle \mathrm{RI}$ by TCD. There is a long-standing controversy about the ability of TCD to accurately reflect volemic CBF (17-19). TCD measures flow velocity in large vessels and makes inferences about "downstream" vascular changes on the assumption that the vessel diameter at the point of insonation remains constant. However, reactivity and diameter changes have been demonstrated in the fetus and newborn $(20,21)$, bringing into question the validity of the TCD technique. Our findings and those of others $(22,23)$ suggest a correlation between measurements made by NIRS and TCD.

To date, the integrity of CPA has been studied mainly through the use of a so-called static approach, which examines the steady-state relationship between $\mathrm{CBF}$ and blood pressure at distinct time points (24-28). CPA is known to be a dynamic system that is influenced by multiple factors (e.g. $\mathrm{CO}_{2}$ tension, hypoxia, drugs) with different mechanisms operating at different rates (29). Our data suggest that CPA is not an "all-ornone" phenomenon but rather a system with fluctuating (dynamic) characteristics. Unlike the static approach, a dynamic approach using a frequency-dependent analysis (e.g. coherence function analysis) is capable of capturing and describing an enormous wealth of CPA data in the time domain. Previous studies have used such a dynamic approach to study CPA using TCD measurements of CBF velocity (12,30-33). Our group previously used coherence function analysis in premature infants and demonstrated that concordant changes between MAP and $\mathrm{HbD}$ ("pressure passive" state) were significantly associated with cerebrovascular lesions (7).

Table 4. Association between ET-CO $\mathrm{C}_{2}$ level and CPA across all data epochs

\begin{tabular}{ccc}
\hline Type of epoch & Early studies $\left[\right.$ mean ET-CO $\left.{ }_{2} \pm \mathrm{SD}(\mathrm{mm} \mathrm{Hg})\right]$ & Late studies $[\mathrm{mean}$ ET-CO \\
\hline Autoregulating epochs* & $\mathrm{SD}(\mathrm{mm} \mathrm{Hg})]$ & $39.0 \pm 5.2(\mathrm{n}=249)$ \\
Pressure-passive epochs $\dagger$ & $35.9 \pm 5.9(\mathrm{n}=296)$ & $41.0 \pm 6.3(\mathrm{n}=43) \S$ \\
\hline
\end{tabular}

$\mathrm{n}$, number of 5-min epochs.

* Autoregulating epochs are 5-minute epochs with no concordant relationship between MAP and HbD (coherence $<0.5$ ).

$\dagger$ Pressure-passive epochs are 5-minute epochs with high concordance between MAP and HbD (coherence $>0.5$ ).

$\ddagger p<0.001$ for autoregulating $v s$ pressure-passive cerebral perfusion.

$\S p=0.04$ for autoregulating $v s$ pressure-passive cerebral perfusion. 
In our study, each 5-min epoch of data was assigned a coherence score; by convention, a coherence score between two signals of 0.5 or above indicates significant concordance between the two signals at that frequency. In our study, 5-min epochs with coherence between $\mathrm{HbD}$ and MAP of 0.5 or above were considered pressure passive. Next we used an index that quantifies disturbed CPA according to its duration over the course of a study, i.e. the percentage of all 5-min epochs that were pressure passive. The relationship between disturbed CPA and subsequent brain injury would be expected to depend on the magnitude of disturbances in blood pressure imposed on the cerebral autoregulatory system. Longer periods of concordance between MAP and HbD may be capable of identifying infants who have disturbed CPA and are at greater risk for brain injury should periods of hemodynamic instability develop. In the current study, most but not all infants had intact or mildly disturbed CPA. When present, a disturbance in CPA was usually evident early in the postoperative course and persisted through the first $20 \mathrm{~h}$ in most cases. The relatively low occurrence of postoperative pressure-passive cerebral circulation in our study may reflect the quality of intraoperative and postoperative treatment of these infants. We recently published data showing a dramatic decrease in the incidence of postoperative neurologic dysfunction at our center (34).

The cerebral pressure autoregulatory system responds to decreases in blood pressure by relaxing the vascular smooth muscle tone, causing vasodilation, thereby maintaining CBF. With a baseline increase in diameter of the resistance arterioles, such as occurs with a lower baseline MAP or increasing circulating $\mathrm{PCO}_{2}, \mathrm{CPA}$ becomes less efficient because of a reduced residual capacity for vasodilation. For this reason, we evaluated the efficiency of CPA in our infants at different levels of mean MAP within the 5-min epochs, as well as at different levels of $\mathrm{P}_{\mathrm{CO}_{2}}$ as induced by ventilator rate changes. We found that increased ambient $\mathrm{PCO}_{2}$ levels were associated with increased odds of autoregulatory failure in the early postoperative period. Previous studies in experimental models and in adult humans have shown similar effects of $\mathrm{PCO}_{2}$ on $\mathrm{CPA}$ (35-40). Because $\mathrm{PCO}_{2}$ is vasoactive in the pulmonary vasculature, changes in $\mathrm{PCO}_{2}$ are used in certain circumstances to adjust pulmonary vascular resistance in infants after cardiac surgery. Our data suggest that hypercarbia may reduce CPA and render the infant more vulnerable to cerebral injury during periods of blood pressure instability.

In this population of critically ill infants, the administration of pharmacologic agents or tilting of the infant solely for the purpose of inducing experimental blood pressure changes is clearly not feasible. We did not attempt to induce changes in baseline blood pressure purely for the sake of the study and relied entirely on "spontaneous" changes in baseline MAP. We found no statistical relationship between baseline MAP levels and the development of a cerebral pressure-passive state in both the early and the late studies. This finding is likely due in large part to the fact that strict hemodynamic management in our ICU successfully maintained baseline blood pressure within normal limits most of the time. This factor also made it impossible for us to address the upper and lower limits of the autoregulatory plateau in these infants. However, the occurrence of superimposed transient oscillations in MAP allowed us to assess CPA during these otherwise stable periods of baseline MAP. In fact, we found that periods of high MAP variability increased the odds of detecting a pressure-passive cerebral state. A number of previous studies have described intrinsic fluctuations of often unclear origin in cerebral hemodynamics and oxygenation $(12,41-46)$. In one report, disturbances in the mechanics of ventilation induced the fluctuations and allowed formulation of an intervention (muscle paralysis) to correct both the ventilatory abnormalities and the fluctuations $(42,43)$. Discussion of these phenomena is beyond the scope of this report.

This study has several potential limitations. Because our studies could begin only once the patient was deemed clinically stable by the attending, some variability in the timing of our measurements was unavoidable and may have introduced some level of bias. However, this effect is likely to be minor. Because the majority of our infants received their arterial lines and endotracheal tubes only in the operating room, we were not able to study reliably their preoperative CPA. Similarly, our recordings could not continue once infants were extubated and arterial lines were discontinued. Second, we attempted to identify perioperative factors (see "Methods") that predisposed to disturbance of CPA in these infants. Among the factors that we analyzed, none was predictive of CPA disturbance, a finding that is likely due to the relatively small number of infants with disturbed CPA in our study and the resulting limitation of statistical power. It is also possible that our study population includes infants who were more hemodynamically stable, thus limiting the generalizability of our findings. Moreover, because the treatment of these infants follows strict treatment guidelines, the limited variability of many clinical factors is so small as to limit significantly the statistical power required to identify antecedents of disturbed CPA in this population.

Finally, as stated above, close clinical monitoring and management of blood pressure in these infants limited the range of blood pressures across which CPA could be measured. However, the goal of this study was to demonstrate the feasibility of this technique for studying CPA in these sick infants rather than to define the limits of their cerebral autoregulatory plateau. Furthermore, the presence of disturbed CPA does not necessarily suggest inevitable subsequent neurologic dysfunction but presumably could serve as an indicator that a given infant is at high risk for ischemic cerebral injury should unstable systemic hemodynamics develop. The importance of our findings will require further studies in the ICU and at follow-up.

In summary, we have demonstrated that continuous measurements of changes in $\mathrm{HbD}$ correlate well with changes in blood flow velocity measured by the more widely used TCD technique. More important, we also demonstrated the feasibility of monitoring and quantifying CPA in sick infants by analyzing the relationship between continuous changes in $\mathrm{HbD}$ and MAP using coherence function analysis. In our population, we found that most but not all infants seemed to have intact CPA during the early postoperative period but that hypercarbia and fluctuating MAP might predispose to a disruption of CPA. 
These findings have implications for the treatment of these infants in the postoperative period.

AcknowledgmentsWe gratefully acknowledge Dr. Joseph J. Volpe for critical review of our manuscript. We also thank Jody Bartlett and Ellen McGrath for research coordination and Shaye Moore and Michelle Mercadante for help in manuscript preparation. Many thanks to the children and their families for participating in this study.

\section{REFERENCES}

1. Bellinger DC, Wypij D, Kuban KC, Rappaport LA, Hickey PR, Wernovsky G, Jonas RA, Newburger JW 1999 Developmental and neurologic status of children at 4 years of age after heart surgery with hypothermic circulatory arrest or low-flow cardiopulmonary bypass. Circulation 100:526-532

2. Majnemer A, Limperopoulos C 1999 Developmental progress of children with congenital heart defects requiring open heart surgery. Semin Pediatr Neurol 6:12-19

3. du Plessis AJ, Newburger JW, Jonas RA, Wessel DL, Wypij D, Tsuji MK, Walter G, Volpe JJ 1995 Cerebral CO2 vasoreactivity is impaired in the early postoperative period following hypothermic infant cardiac surgery. [abstract] Eur J Neurol 2:68A

4. Lassen NA 1959 Cerebral blood flow and oxygen consumption in man. Physiol Rev 39:183-233

5. Tsuji M, duPlessis A, Taylor G, Crocker R, Volpe JJ 1998 Near infrared spectroscopy detects cerebral ischemia during hypotension in piglets. Pediatr Res 44:591-595

6. Soul JS, Taylor GA, Wypij D, DuPlessis AJ, Volpe JJ 2000 Noninvasive detection of changes in cerebral blood flow by near-infrared spectroscopy in a piglet model of hydrocephalus. Pediatr Res 48:445-449

7. Tsuji M, Saul JP, du Plessis AJ, Eichenwald E, Sobh J, Crocker R, Volpe JJ 2000 Cerebral intravascular oxygenation correlates with mean arterial pressure in critically ill premature infants. Pediatrics 106:625-632

8. du Plessis AJ, Jonas RA, Wypij D, Hickey PR, Riviello J, Wessel DL, Roth SJ, Burrows FA, Walter G, Farrell DM, Walsh AZ, Plumb CA, del Nido P, Burke RP, Castaneda AR, Mayer JE Jr, Newburger JW 1997 Perioperative effects of alpha-sta versus $\mathrm{pH}$-stat strategies for deep hypothermic cardiopulmonary bypass in infants. J Thorac Cardiovasc Surg 114:991-1001

9. du Plessis AJ 1995 Near-infrared spectroscopy for the in vivo study of cerebral hemodynamics and oxygenation. Curr Opin Pediatr 7:632-639

10. Soul JS, du Plessis AJ 1999 New technologies in pediatric neurology. Near-infrared spectroscopy. Semin Pediatr Neurol 6:101-110

11. Reinhard M, Muller T, Guschlbauer B, Timmer J, Hetzell A 2003 Transfer function analysis for clinical evaluation of dynamic cerebral autoregulation-a comparison between spontaneous and respiratory-induced oscillations. Physiol Meas 24:27-43

12. Zhang R, Zuckerman JH, Giller CA, Levine BD 1998 Transfer function analysis of dynamic cerebral autoregulation in humans. Am J Physiol 274:H233-H241

13. Saul JP, Rea RF, Eckberg DL, Berger RD, Cohen RJ 1990 Heart rate and muscle sympathetic nerve variability during reflex changes of autonomic activity. Am J Physiol 258:H713-H721

14. Gemelli M, Manganaro R, Marni C, De Luca F 1990 Longitudinal study of blood pressure during the 1st year of life. Eur J Pediatr 149:318-320

15. Pryds O, Greisen G, Skov LL, Friis-Hansen B 1990 Carbon dioxide-related changes in cerebral blood volume and cerebral blood flow in mechanically ventilated preterm neonates: comparison of near infrared spectrophotometry and 133 Xenon clearance. Pediatr Res 27:445-449

16. Wyatt JS, Edwards AD, Cope M, Delpy DT, McCormick DC, Potter A, Reynolds EO 1991 Response of cerebral blood volume to changes in arterial carbon dioxide tension in preterm and term infants. Pediatr Res 29:553-557

17. Kontos H 1989 Validity of cerebral blood flow calculations from velocity measurements. Stroke 20:1-3

18. Newell DW, Aaslid R, Lam A, Mayberg TS, Winn HR 1994 Comparison of flow and velocity during dynamic autoregulation testing in humans. Stroke 25:793-797

19. van der Linden J, Wesslen O, Ekroth R, Tyden H, von Ahn H 1991 Transcranial Doppler-estimated versus thermodilution-estimated cerebral blood flow during cardiac operations. Influence of temperature and arterial carbon dioxide tension. J Thorac Cardiovasc Surg 102:95-102
20. Gilbert RD, Pearce WJ, Ashwal S, Longo LD 1990 Effects of hypoxia on contractility of isolated fetal lamb cerebral arteries. J Dev Physiol 13:199-203

21. Longo LD, Pearce WJ 1991 Fetal and newborn cerebral vascular responses and adaptations to hypoxia. Semin Perinatol 15:49-57

22. Smielewski P, Kirkpatrick P, Minhas P, Pickard JD, Czosnyka M 1995 Can cerebrovascular reactivity be measured with near-infrared spectroscopy? Stroke 26:22852292

23. Smielewski P, Czosnyka M, Pickard JD, Kirkpatrick P 1997 Clinical evaluation of near infrared spectroscopy for testing cerebrovascular reactivity in patients with carotid artery disease. Stroke 28:331-338

24. Jorch G, Jorch N 1987 Failure of autoregulation of cerebral blood flow in neonates studied by pulsed Doppler ultrasound of the internal carotid artery. Eur J Pediatr 146:468-472

25. Cowan F, Eriksen M, Wertheim DPF 1989 Regulation of cerebral blood flow in the newborn preterm human infant. [abstract] J Physiol 417:13P

26. Pryds O, Greisen G, Lou H, Friis-Hansen B 1990 Vasoparalysis associated with brain damage in asphyxiated term infants. J Pediatr 117:119-125

27. Bouma GJ, Muizelaar JP, Bandoh K, Marmarou A 1992 Blood pressure and intracranial pressure-volume dynamics in severe head injury: relationship with cerebral blood flow. J Neurosurg 77:15-19

28. Tiecks FP, Lam AM, Aaslid R, Newell DW 1995 Comparison of static and dynamic cerebral autoregulation measurements. Stroke 26:1014-1019

29. Vavilala MS, Lee LA, Lam AM 2002 Cerebral blood flow and vascular physiology. Anesthesiol Clin North Am 20:247-264

30. Giller CA 1990 The frequency-dependent behavior of cerebral autoregulation. Neurosurgery $27: 362-368$

31. Panerai RB, Kelsall AW, Rennie JM, Evans DH 1996 Analysis of cerebral blood flow autoregulation in neonates. IEEE Trans Biomed Eng 43:779-788

32. Panerai RB, Rennie JM, Kelsall AW, Evans DH 1998 Frequency-domain analysis of cerebral autoregulation from spontaneous fluctuations in arterial blood pressure. Med Biol Eng Comput 36:315-322

33. Portnoy HD, Chopp M, Branch C, Shannon MB 1982 Cerebrospinal fluid pulse waveform as an indicator of cerebral autoregulation. J Neurosurg 56:666-678

34. Menache CC, du Plessis AJ, Wessel DL, Jonas RA, Newburger JW 2002 Current incidence of acute neurologic complications after open-heart operations in children. Ann Thorac Surg 73:1752-1758

35. Haggendal E, Johansson B 1965 Effects of arterial carbon dioxide tension and oxygen saturation on cerebral blood flow autoregulation in dogs. Acta Physiol Scand Suppl 258:27-53

36. Harper AM 1966 Autoregulation of cerebral blood flow: influence of the arterial blood pressure on the blood flow through the cerebral cortex. J Neurol Neurosurg Psychiatry 29:398-403

37. Paulson OB, Olesen J, Christensen MS 1972 Restoration of autoregulation of cerebral blood flow by hypocapnia. Neurology 22:286-293

38. Aaslid R, Lindegaard KF, Sorteberg W, Nornes H 1989 Cerebral autoregulation dynamics in humans. Stroke 20:45-52

39. Newell DW, Weber JP, Watson R, Aaslid R, Winn HR 1996 Effect of transient moderate hyperventilation on dynamic cerebral autoregulation after severe head injury. Neurosurgery 39:35-43; discussion 43-44

40. Smielewski P, Czosnyka M, Kirkpatrick P, McEroy H, Rutkowska H, Pickard JD 1996 Assessment of cerebral autoregulation using carotid artery compression. Stroke 27:2197-2203

41. Obrig H, Neufang M, Wenzel R, Kohl M, Steinbrink J, Einhaupl K, Villringer A 2000 Spontaneous low frequency oscillations of cerebral hemodynamics and metabolism in human adults. Neuroimage 12:623-639

42. Perlman JM, McMenamin JB, Volpe JJ 1983 Fluctuating cerebral blood-flow velocity in respiratory-distress syndrome. Relation to the development of intraventricular hemorrhage. N Engl J Med 309:204-209

43. Perlman JM, Goodman S, Kreusser KL, Volpe JJ 1985 Reduction in intraventricular hemorrhage by elimination of fluctuating cerebral blood-flow velocity in preterm infants with respiratory distress syndrome. N Engl J Med 312:13531357

44. Fujii K, Heistad DD, Faraci FM 1990 Ionic mechanisms in spontaneous vasomotion of the rat basilar artery in vivo. J Physiol (Lond) 430:389-398

45. Diehl RR, Linden D, Lucke D, Berlit P 1998 Spontaneous blood pressure oscillations and cerebral autoregulation. Clin Auton Res 8:7-12

46. von Siebenthal K, Beran J, Wolf M, Keel M, Dietz V, Kundu S, Bucher HU 1999 Cyclical fluctuations in blood pressure, heart rate and cerebral blood volume in preterm infants. Brain Dev 21:529-534 